\title{
INVESTIGATION ON SINGLE POINT INCREMENTAL FORMING PROCESS CONSIDERING VARIOUS TOOL PATH DEFINITIONS
}

\author{
MOHANRAJ MURUGESAN ${ }^{1}$, MUHAMMAD SAJJAD ${ }^{2}$ \& DONG WON JUNG ${ }^{3}$ \\ ${ }^{1 \& 2}$ Research Scholar, Department of Mechanical Engineering, Jeju National University, Jeju Island, South Korea \\ ${ }^{3}$ Professor, Department of Mechanical Engineering, Jeju National University, Jeju Island, South Korea
}

\begin{abstract}
Single point incremental forming (SPIF) has a great contribution in industrial practice, because the desired parts such as the small-batch production and prototype products can be manufactured just by changing the punch tools and supports. The sheet is formed into desired part using punch tools by producing local plastic deformations at the contact locations based on designed tool paths. This research work is aimed to investigate the formability of SPIF process over the numerical simulations in terms of various geometries and tool paths with varied constant depth, tool radius and feed rate test conditions. The mechanical properties of an aluminum alloy were considered and incorporated into the finite element (FE) code and further, the procedure to perform the forming process was modeled in LS-DYNA tool. To reduce computational time and eliminate the inconsistent results, the symmetry boundary conditions for symmetrical shapes were exploited and discussed. Thereafter, results achieved from the numerical simulations were compared with the desired part to make sure that the suitable FE model was developed. Discussion on the thickness distributions in terms of thinning area, thinning location and its size in percentage are presented. The thickness reduction in both geometries indicates that thinning occurs uniformly in the wall region and small fluctuation noticed near the tool retraction location. In detail, a bending region was observed with continuous thickness reduction and the desired geometry was accomplished from the base plate configuration by controlling the stretching deformation at the start of forming process.
\end{abstract}

KEYWORDS: Single Point Incremental Forming, Finite Element Model, Thinning Location, Base Plate Configuration \& Symmetry Boundary Conditions

Received: Sep 14, 2019; Accepted: Oct 04, 2019; Published: Nov 06, 2019; Paper Id.: IJMPERDDEC201944

\section{INTRODUCTION}

Aluminium alloys have been the best choice material for the manufacturing of aircraft and automobile components even though these days, the materials such as titanium and composite materials are widely utilized for the industrial construction. It is obvious that most of the airframe and automobile components are made from aluminium alloy materials because of its advantages such as low density, appropriate material properties, easy to manufacture and cost effective [1]. Over the past few years, the manufacturing methods for the specific applications are in development without compromising the customer requirements. Another main thing to remember that the developed method should be useful for manufacturing any new products and also the modifications have to be made easily if necessary in the product design. Sheet metal-forming process like roll forming, deep drawing, stamping and incremental sheet forming, is one of the major and complex manufacturing process in automobile and aerospace industries. Importantly, it requires an extensive knowledge related to the manufacturing such as deformation mechanism associated with forming and the 
process parameters like desired part design, die and punch design, holder force etc. Moreover, having the profitable design without any defects can leads to best outcome in terms of production quality, mass manufacturing and also can keep the industries to be stable economic wise. For this purpose, the finite element (FE) method can be useful to simulate the forming process in the early stages of design process and significantly, after lots of trail running, the favourable forming design can be achieved eventually, however it requires more computational time and effort. Notably, for the past decade, a new manufacturing approach called single point incremental forming (SPIF) is widely employed by several researchers to produce the hollow parts as shown in Figure 1(a). The main advantages of this method is its die-less processing which, the sheet is manufactured into the final desired shape by means of local plastic deformations and moreover, the entire process can be controlled using the computer numerical control (CNC) milling machine. In detail, the punch tool attached to the machine is continuously drawn into the metal sheet based on the tool path designed for the final part. In addition, the process parameters such as feed rate, spindle speed, friction coefficient, tool shape, sheet temperature and tool path, has to be considered significantly in order to control the dimension accuracy in the complex shapes.

Several authors recognized that the formability of sheet metal from the incremental sheet forming (ISF) method is much more effective than the conventional deep drawing and the stamping process, but they also stated that there is a loss of accuracy in the final manufactured parts. To overcome these difficulties, extensive number of research work has been conducted related to ISF process considering various methods and techniques. Gipiela et al.[2] examined SPIF process using finite element method in order to investigate the forming limits of high strength low alloy steel material. In addition, they were compared the results achieved by simulation and experiments with Nakajima deep drawing results. It was stated that SPIF outweigh the forming limits of Nakajima limit and also concluded that the strain obtained from the concentric profile is minimum in the formed parts. Malwad et al.[3], Manish Oraon[4] et al. and Suresh Kurra et al.[5] were conducted enough number of experiments to discuss the surface quality of formed products in terms of working parameters such as step size, forming angle, tool diameter, feed rate and lubricant type. From the outcome, Manish Oraon et al.[4] and Suresh Kurra et al.[5] developed a surface roughness prediction models using artificial neural networks, support vector regression and genetic programming. Moreover, the optimum parameters obtained from the prediction model were tested against the experiments and the results found to be adequate with the experimental data. The tool path influence on formability and localized thinning of AA7075-O material in SPIF process was investigated using both experiments and numerical models by Erika Salem et al.[6] and Neto et al.[7]. They developed and analysed SPIF process with the help of finite element model in terms of strain, stress and thickness distribution in the formed parts. The residual stresses in both circumferential and meridional directions were found to be positive in the inner skin and negative in the outer skin and in addition, the stresses develops predominantly along the circumferential direction because of the geometrical restrictions. Adrian et al. [8] also conducted SPIF study to investigate the influence of tool path on main strains distribution and relative thinning and forces using optical methods. For this purpose, they considered various tool paths with constant vertical step and angular constant step etc. In the forming process, it is necessary to know the magnitude of forces in order to choose the punch tool and also to make sure that the equipment available has a potential to perform the forming without any limitations. Jeswiet et al.[9] inspected the force magnitudes in the 
process, in order to develop an appropriate model for AA-3003 material considering cones and truncated pyramid shapes.
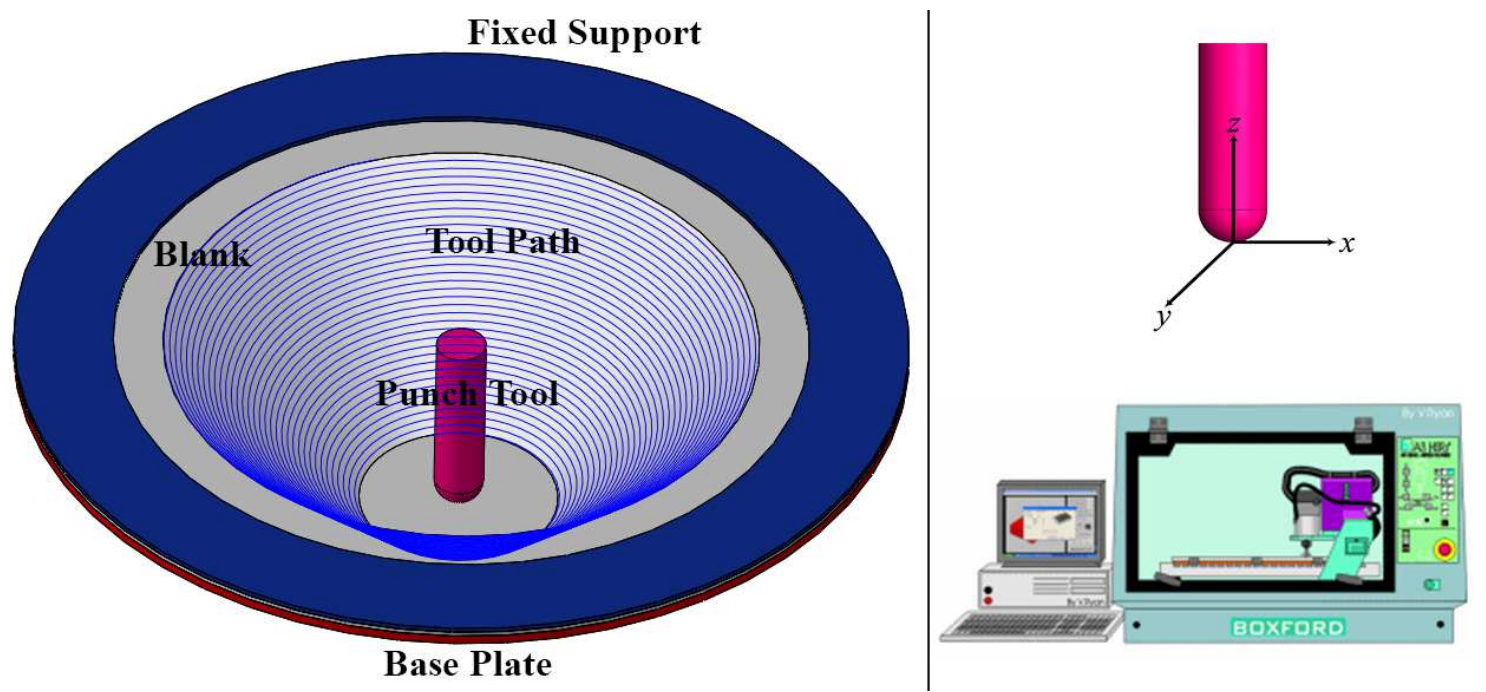

(a) Single Point Incremental Sheet Forming Principle.

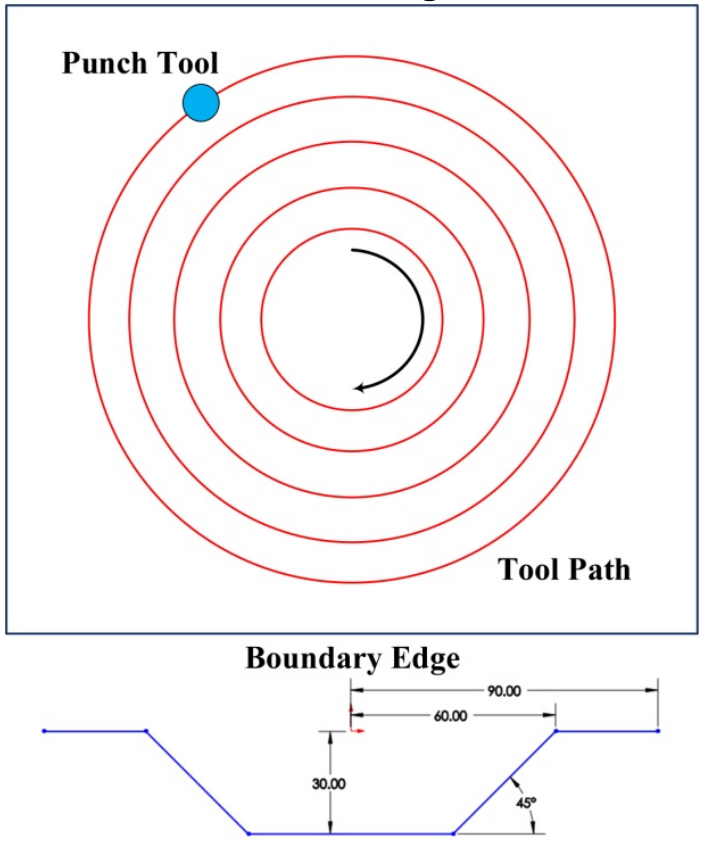

(b) Tool Path Contour for Truncated Cone

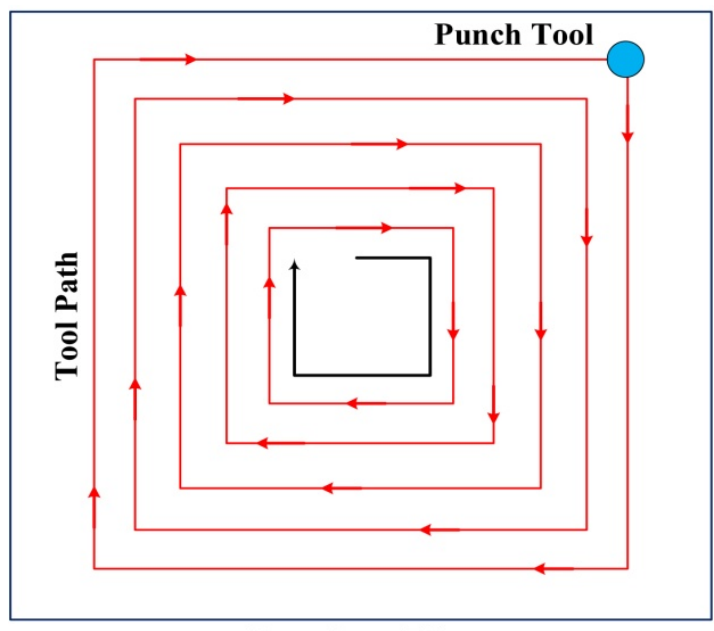

Boundary Edge

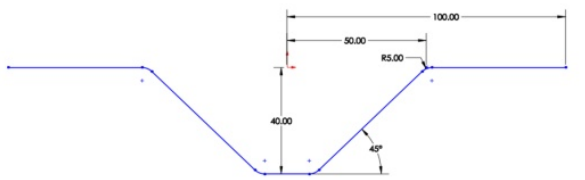

(c) Tool Path Contour for Truncated Pyramid

Figure 1: Single Point Incremental Sheet Forming Procedures.

Generally, the material formability is greatly affected by the process parameters such as spindle speed, feed rate, and tool diameter, lubricant and moreover tool path adopted. Markan day et al.[11] investigated the formability issues more in detail to achieve better formability and for this purpose, the influence of process parameters were included into the forming process using the helical tool path strategy on the commercial pure aluminium material. Further, the formed products were tested for spring back, thinning percentage and maximum forming depth. Experiments were conducted by adjusting the tool speed and the feed rate. At one case, the tool speed is increased and the feed rate kept constant, from the outcome, the forming depth and thinning were found to be increased and vice versa in the other case. Overall, spring back was found to be maximum when the feed rate is higher rather than the tool speed. One more important investigation 
required in the forming process is obtaining the geometric accuracy after forming process. Denis Daniel et al.[12] researched the geometrical accuracy via an experimental survey to examine the influence of the support force and the support angle. Their influences are discussed in detail with the help of various experimental results from SPIF process. Naranjo et al.[13] explored the advantage of finite element tools by investigating various shapes of titanium ASTM B-265. They stated that ANSYS tool can simulate SPIF process more effectively when finer elements are considered during the meshing procedure. Though, the numerical model can be helpful to understand the deformation mechanism and the influence variables in each step in terms of stress and strain distribution during the forming process. Likewise, Memicoglu et al. [15] exploited the numerical model for suggesting the best approach to reduce the computational time, by adopting the proper meshing procedure and symmetric boundary conditions. From the results, they concluded that the developed numerical model can reduce the computational time up to 24 times with a maximum error of $6 \%$ in the final product.

The aim of this present work was to investigate the formability of aluminium alloy material considering various tool path strategies. The shapes like a truncated cone and truncated pyramid geometries were considered in order to analyse SPIF process as depicted in Figures. 1(b) and 1(c). The mechanical properties of aluminium alloy were obtained from the room temperature tensile test and also the properties were incorporated into LS-DYNA commercial tool by the material card to define the material ductility behaviour. Besides, the various tool paths for both geometries were designed in CATIA/CAM software with different step size and simultaneously, the generated coordinates were used for conducting SPIF numerical simulation. The numerical results are compared with the expected geometry results to verify the model usefulness. In addition, the model symmetric conditions are exploited to reduce the computational time and the simulated results are discussed against the full numerical model.

\section{SPIF FINITE ELEMENT MODEL}

The room temperature tensile test procedures were used to characterize the ductility behaviour of a work material. Initially, the flat specimens were machined from an aluminium alloy sheets according to the KSB0802 standards and tested at room temperatures as shown in Figure 2(a). The load-displacement curves were converted into stress-strain (SS) curves adopting the simple tensile test equations. The obtained engineering and true SS curves are depicted in Figure 2(a). The SS curves were approximated by the Hollomon power law, Eq.(1), in order to represent the material strain hardening behaviour.

$$
\sigma=K \varepsilon^{n}
$$

In Eq.(1), $\sigma, K, \varepsilon$ and $n$ are true stress, strength coefficient, true strain and strain hardening coefficient, respectively. In order to understand the development of process and the behaviour of isotopic material, the virtual tensile test was modeled in LS-DYNA by inputting the Hollomon model constants and the mechanical properties of an aluminium alloy as outlined in Table 1 . The numerical result displays that properly defining the properties in a material card can produce accurate results in terms of the material behaviour at each stage and damage location as compared in Figure 2(a).

Table 1: Mechanical Properties of Aluminium Alloy (Al6061) Material

\begin{tabular}{|c|c|c|c|c|c|}
\hline $\boldsymbol{\rho}\left(\mathbf{K g} / \mathbf{m}^{3}\right)$ & $\mathbf{E}(\mathbf{G P a})$ & $\boldsymbol{\sigma}_{\mathbf{Y}}(\mathbf{M P a})$ & $\mathbf{v}$ & $\mathbf{K}(\mathbf{M P a})$ & $\mathbf{n}$ \\
\hline 2710 & 68.6 & 271 & 0.33 & 514.8 & 0.1811 \\
\hline
\end{tabular}

A truncated cone and pyramid shapes with $45^{\circ}$ wall angle were designed in CATIA/CAD using the dimensions illustrated in Figures 1 (b) and 1(c). A corner radius at both geometries was chosen based on the tool diameter in order to 
maintain the shape accuracy while forming. Moreover, it is obvious that the material properties and the tool path contour are the most important inputs for SPIF process. So after designing the model, in the next step, the tool path was generated in CATIA/CAM commercial package including various step size increments to investigate the formability of SPIF process. A tool path was generated from $0.5 \mathrm{~mm}$ to $3 \mathrm{~mm}$ with a tool radius of $2 \mathrm{~mm}$ and a feed rate of $1000 \mathrm{~mm} / \mathrm{min}$ for both geometries and to give an illustration the contour paths are plotted in Figures 1 (b) and 1(c).

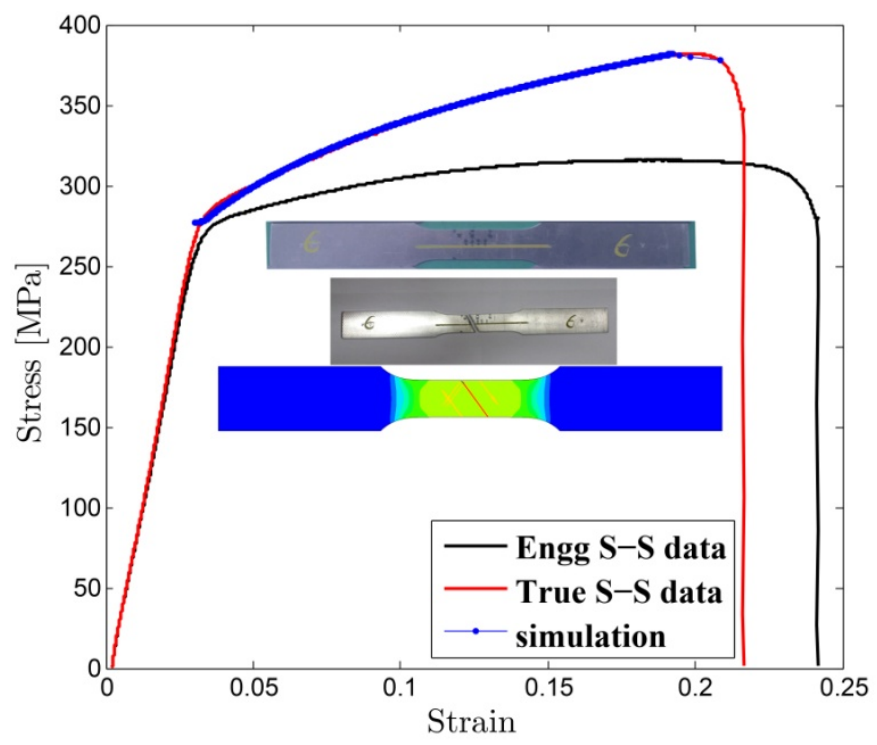

(a) Stress-Strain Curves (Al6061-T6)

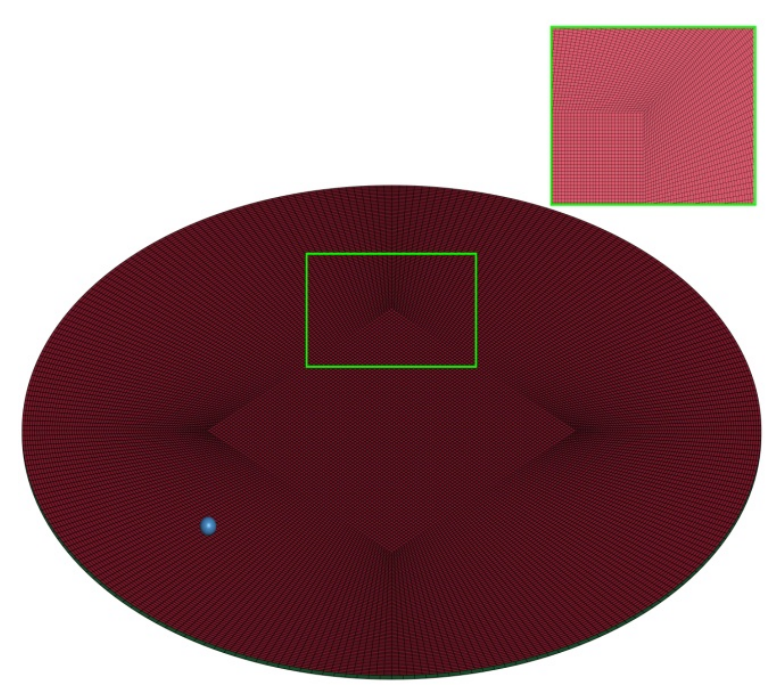

(b)Finite Element Model.

Figure 2: (a) Stress-Strain Curves (Al6061-T6); (b) Finite Element Model.

Likewise, the FE model of SPIF process was developed as depicted in Figure 2(b) and the mechanical properties achieved from the tensile test were incorporated using a material card (MAT18). Both blank and punch tool were meshed fine enough to control accuracy and also to reduce computational time. The friction coefficient was considered close to zero and as the sheet thickness was considerably minimum, the shell element formulation (ELFORM2) with 5 integration points is used for solving SPIF problem. As well as a contact between blank and tool was defined using forming one way surface to surface with soft option 4 to control the penetrations during forming process and the punch tool movement was defined using an imported tool path coordinates from the commercial CAM package.

After SPIF process, the material thinning behaviour can be estimated from the sine law in terms of final wall thickness $\left(t_{1}\right)$ using a relationship between the wall angle $(\alpha)$ and the initial wall thickness $\left(t_{0}\right)$ as expressed below [14]:

$$
t_{1}=t_{0} \sin \alpha
$$

However, Young et al.[17] and Ambrogio et al. [18] showed that the sine law prediction accuracy was found to show some deviation, to put it in another way, the thickness was varying in the formed part along the wall angle. The thickness estimation was less than the sine law prediction in Young et al. [17] research work and they concluded that it may be due to an over spinning condition in shear forming. Similarly, in Ambrogio et al. [18] work, close to the sheet perimeter, the thickness was determined to be higher than the prediction as well as lower in the remaining profile location. 


\section{SPIF SIMULATION RESULTS}

Using previous section details [2], SPIF process simulation was modeled for the working geometries. Before move into the actual solution, enough number of runs were tried in order to obtain a large time step to minimize the computational time. Lately, it was identified from an outcome that by choosing proper mass scaling value (Equation (3)), the simulation run time reduction can be achieved.

$$
\rho_{i}=\frac{\left(\Delta t_{\text {specified }}\right)^{2} \cdot E}{l_{i}^{2} \cdot\left(1-v^{2}\right)} \quad i \text { for element number }
$$

In this work, positive value of time step size for mass scaled solution (DT2MS) was used, so all elements were allocated to have same time step size by adjusting the density $(\rho)$.

The numerical result of SPIF process for truncated cone shape is depicted in Figure 3(a). To validate the FE model, the cross section coordinates were obtained by creating a path along the longitudinal direction as shown in Figure 3(a) and then the numerical section was compared with the real section coordinates as illustrated in Figure 3(b). This proves that the numerical model can be utilized for further discussion about SPIF process considering various process parameters. Figure 3(b) is evident that the blank experiences bending at early stage of forming and that results in loosing accuracy in the blank final shape. This is because, during forming, the blank involves both bending and stretching deformations and in other words, due to the punch tool motions, the blank expose to bending deformation because of tool compression and stretching deformation, as a result of tool radial motion. This supports the idea that by placing an extra support under the blank can control the bending at the early stage of forming. Notably, the result with base plate (BP) configuration indicates that the initial bending issue can be solved as shown in Figure 3(c). In addition, the various tool path generations are tested and compared against base design and found that as step size goes higher the geometry accuracy tends to be lower with occurrence of maximum pillow effect at un deformed location.

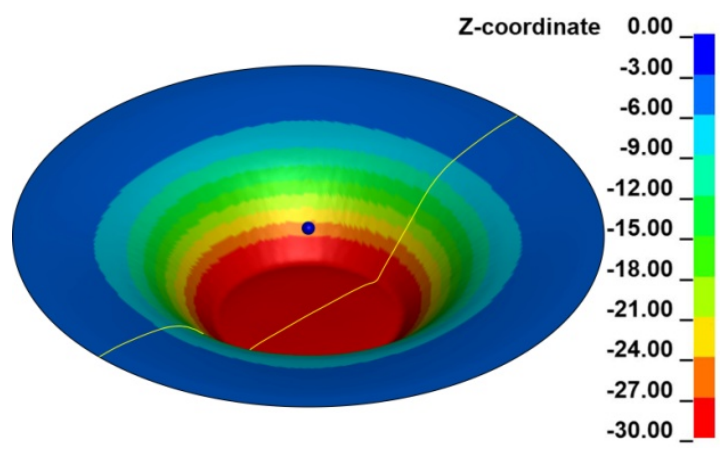

(a) Formed Truncated Cone Profile

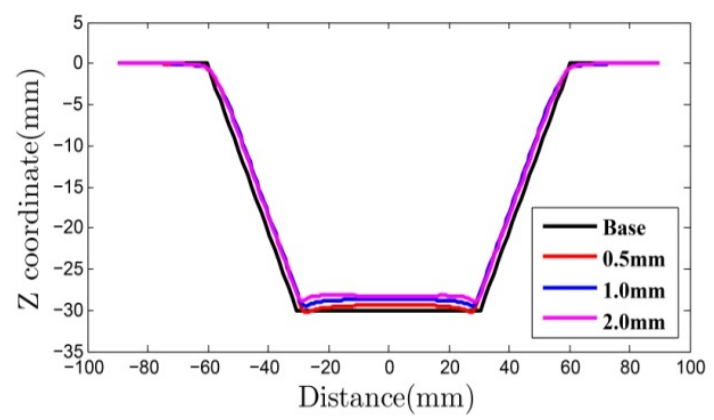

(c) Profile Comparison (with BP)

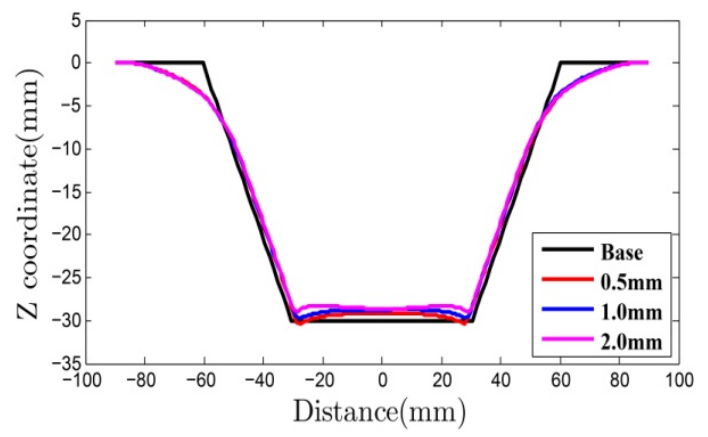

(b) Profile Comparison (without BP)

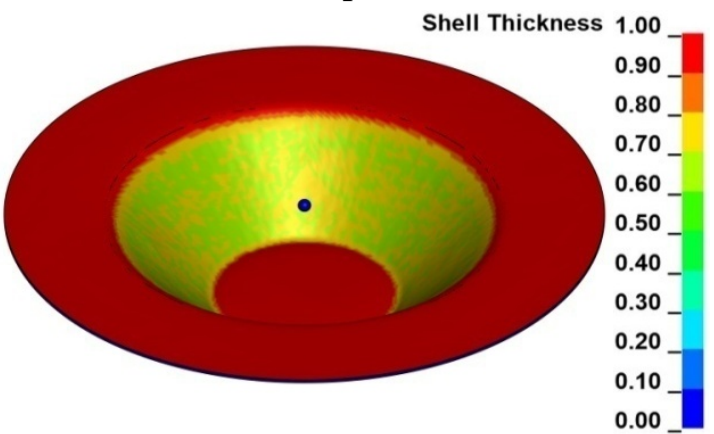

(d) Thinning Behaviour (with BP)

Figure 3: SPIF Numerical Results of Truncated Cone Geometry. 
Likewise, the thickness distribution in formed profile was plotted along the cut section as depicted in Figure 4(a). The thinning quantity in terms of both sum and percentage in tested contour paths shows that the difference is almost close to each other as outlined in Table 2. From base geometry, it can be seen that the model could be partitioned into two sections: section that intent to deform and another section that not intent to deform. Based on this, the sine law used to develop a thickness variation for these sections and plotted against the numerical result in figure 4(a). From simulation results, it can be seen that at start, the blank experiences bending deformation in which the plastic deformation is not expected to happen and it is because of the tool compression and the support distance. After that the thinning phenomenon found to be stable and showed steady progressive but at the tool retraction region, the thickness variation displayed a little oscillations. Compared with the sine law estimation, the thinning occurs almost inside the theoretical prediction and only $0.033 \mathrm{~mm}$ is higher than the sine law prediction. Coupled with base plate configuration, the initial bending issues are significantly minimized and also the thickness variation happens to be within the sine law prediction with small error quantity as shown in figure 4(b). Similarly, the plastic strain distributions are drawn in terms of quantification and contour verifications as plotted in Figures 4(d) and 4(e) and from figure 4(e), it is realised that uniform strain all over the formed shape was identified. Furthermore, due to the close movement of punch tool between paths, the strain value is found to be higher at $0.5 \mathrm{~mm}$ step size whereas the lower strain value occurred at $2 \mathrm{~mm}$ step size because of large gap between paths. As a result of interaction between blank and punch tool, the stress fluctuation is occurred in the formed profile as shown in figure 4(f). Table 2 is evident that the milling time is increasing gradually when the contour tool step down size is decreasing. These discussions prove that the developed SPIF numerical model can be used for modelling a truncated pyramid shape incremental sheet forming process.

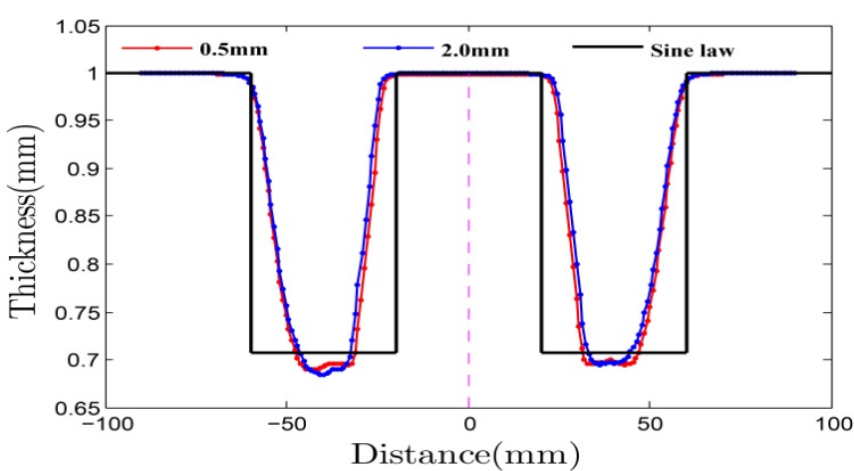

(a) Thickness Distribution (without BP) \% Thickness Reduction- based on current z-strain

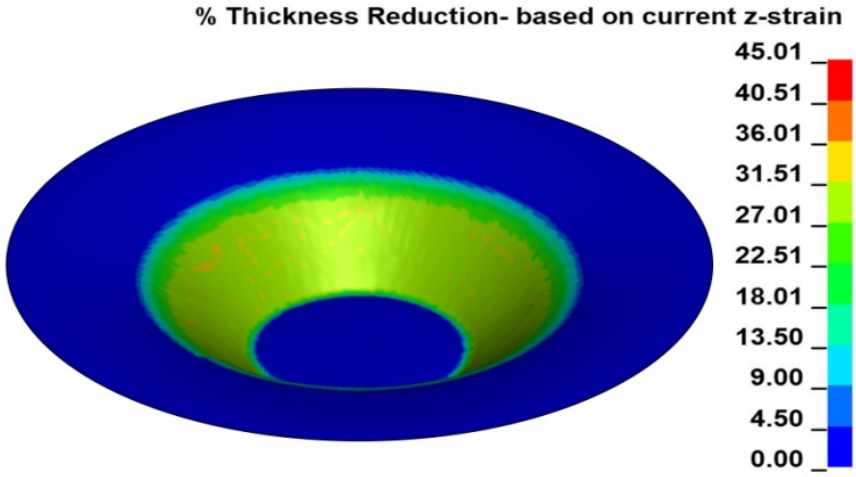

(c) Thickness Reduction Contour in \%

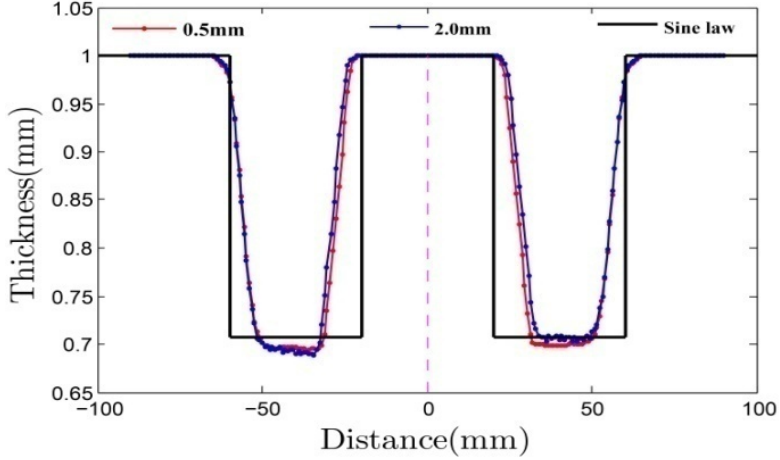

(b) Thickness Distribution (with BP)

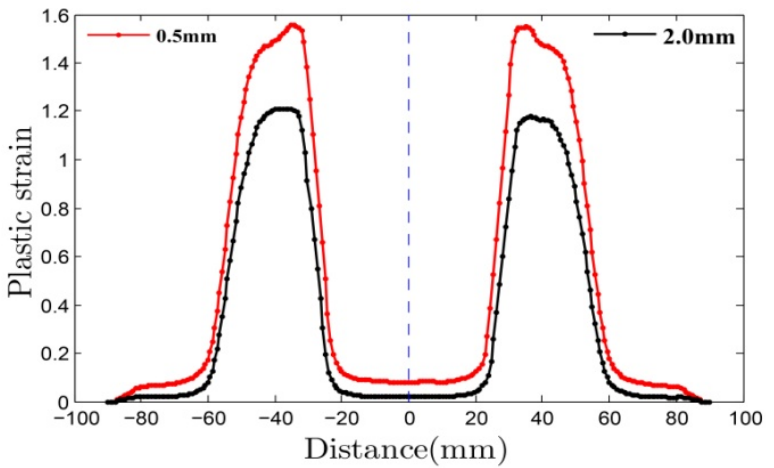

(d) Plane Strain Distribution (without BP) 


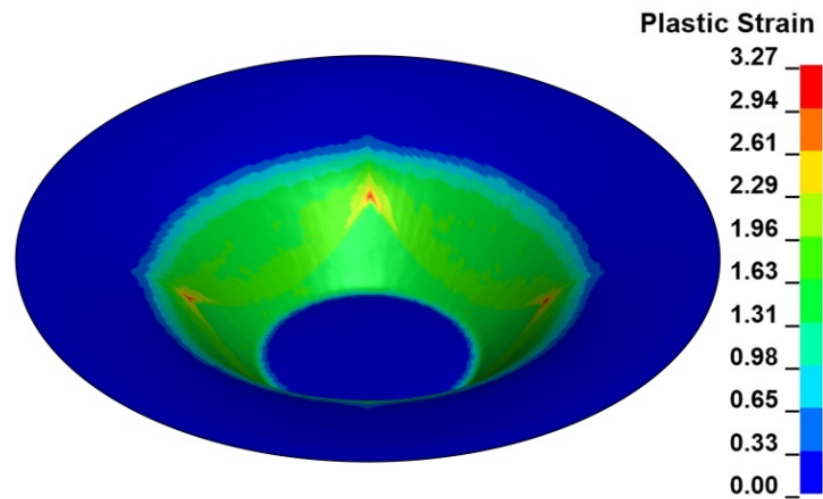

(e) Plastic Strain Contour (without BP)

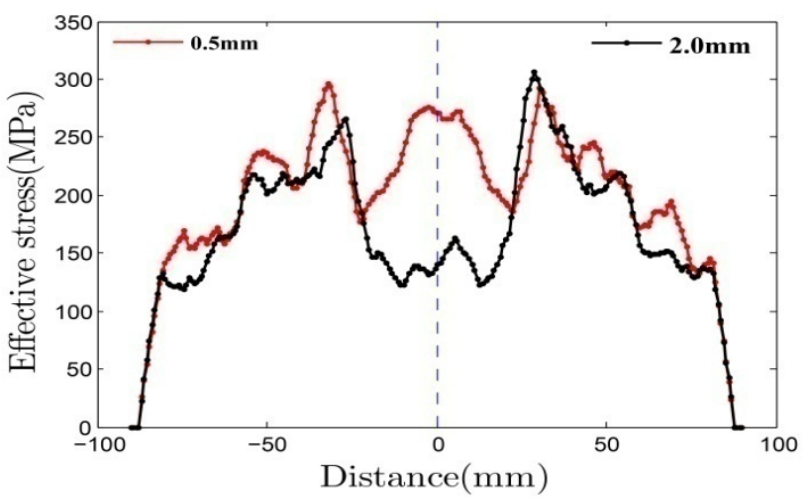

(f) Effective Stress Distribution (without BP)

Figure 4: SPIF Simulation Results of Truncated Cone in Terms of Strain, Stress and Thickness Distributions.

Based on discussions presented in previous paragraph, the FE model of SPIF process for truncated pyramid geometry was established using three types of generated contour paths. Using a similar approach as aforementioned, the numerical geometry coordinates from the cut-section as shown in figure 5(a) are compared with the base cad geometry. Figure 5(b) displays that the blank experienced bending at start of forming process due to tool compression near that region while forming process begins as it also can be seen figure 3(b). From previous experience, this result indicates that the bending problem can be solved by putting extra support at the region where the deformation was not planned and figure 5(c) proves that the stable deformation can be achieved as proposed. However, the opposite is seen in the geometrical accuracy as the simulated results showed a considerable variation from designed shape with the maximum error varies from 6 to $25 \%$ in the selected locations. The deviation may be due to the limitations of forming depth and wall angle in SPIF process for forming a truncated cone shape and to make this point as solid conclusion, it requires experimental validation, and which can be done eventually near the future in our research work. In addition, Figure 5(d) shows the thickness variation along the forming depth and explains a uniform and symmetric behaviour of formed part.

Table 2: SPIF Numerical Conditions and Results Comparison of Truncated Cone

\begin{tabular}{|c|c|c|c|c|c|}
\hline Tool Radius & Feed Rate & Step Size & Thickness (max) & Thickness (\%) & Milling Time \\
\hline \multirow{8}{*}{$2 \mathrm{~mm}$} & \multirow{8}{*}{$1000 \mathrm{~mm} / \mathrm{min}$} & \multirow{2}{*}{$0.5 \mathrm{~mm}$} & 0.6896 & 31.063 & \multirow{2}{*}{$18 \min 42 \mathrm{~s}$} \\
\hline & & & 0.6944 & 30.448 & \\
\hline & & \multirow{2}{*}{$1.0 \mathrm{~mm}$} & 0.6821 & 31.801 & \multirow{2}{*}{$9 \min 25 s$} \\
\hline & & & 0.6906 & 31.057 & \\
\hline & & \multirow{2}{*}{$2.0 \mathrm{~mm}$} & 0.6837 & 31.632 & \multirow{2}{*}{$4 \min 47 s$} \\
\hline & & & 0.6878 & 31.326 & \\
\hline & & \multirow{2}{*}{$3.0 \mathrm{~mm}$} & 0.6736 & 32.677 & \multirow{2}{*}{$3 \min 21 s$} \\
\hline & & & 0.6811 & 32.015 & \\
\hline
\end{tabular}




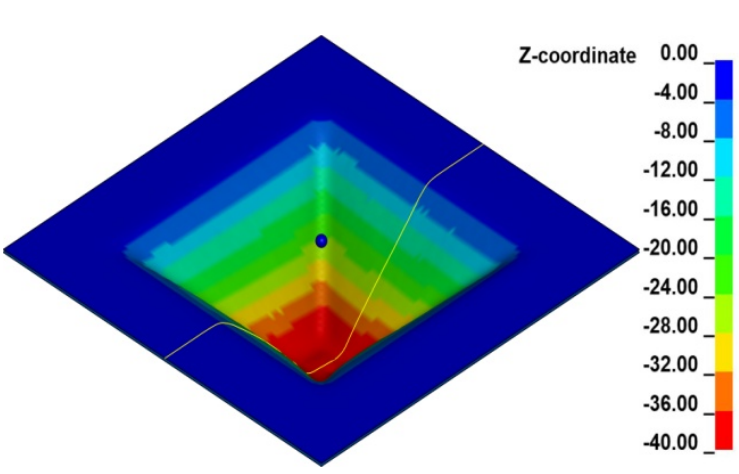

(a) Formed Truncated Pyramid Profile

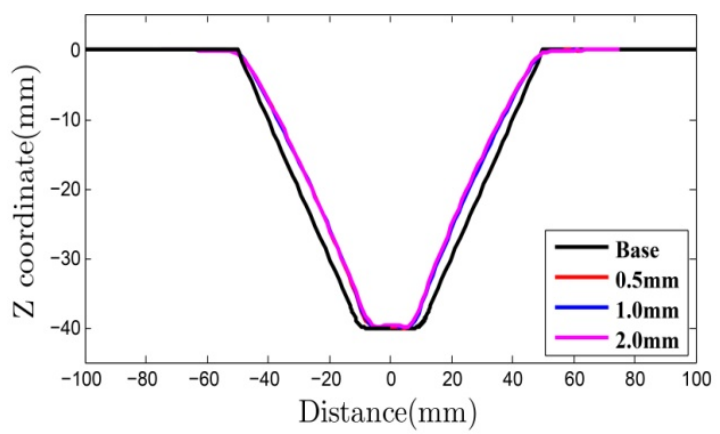

(c) Profile Comparison (with BP)

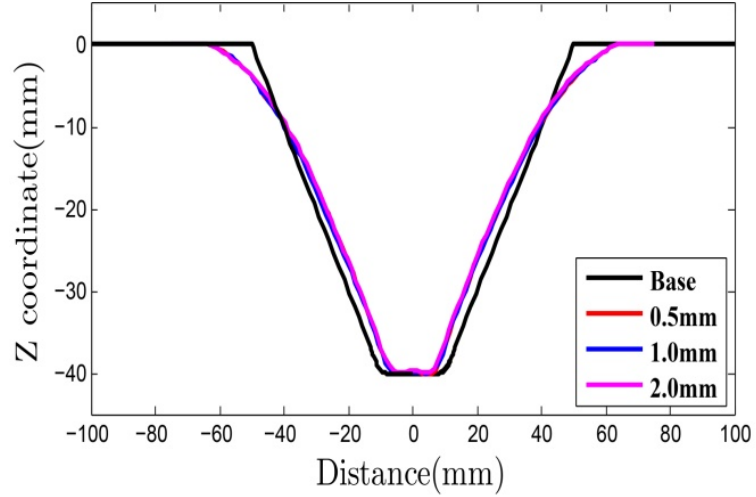

(b) Profile Comparison (without BP)

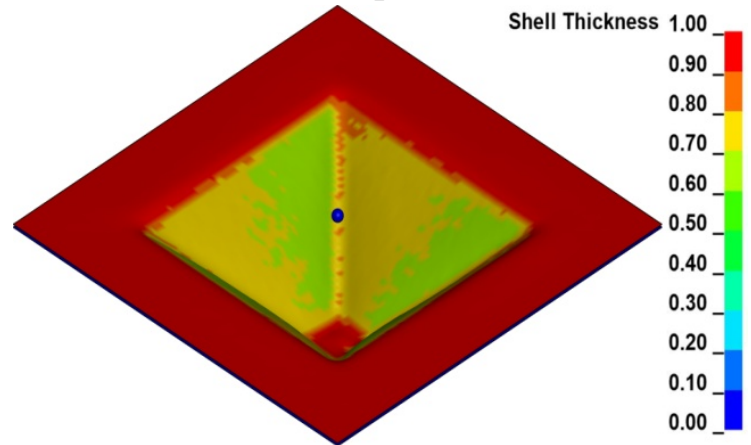

(d) Thinning Behaviour (with BP)

Figure 5: SPIF Numerical Results of Truncated Pyramid Shape.

In comparison with the simulated results and the sine law as illustrated in Figures 6(a) and 6(b), it is noted that the thickness variation in rolling direction flawlessly lies along the sine law prediction but at the same time in transverse direction, it under predicts the thinning behaviour and showed small fluctuated variations. It is important to mention that during forming process, the blank reacts with the punch tool in terms of two deformations: firstly, stretching to cause the deformation in radial direction and secondly, tool compression to produce the deformation in downward direction by stepdown procedure to achieve desired product. So clearly, because of stretching in transverse direction, the blank experiences both twisting and excessive thinning in that region during forming process. Figures 6(c) and 6(d) are evident that the plastic strains at near the support region are close to zero and indicates that the deformation is limited in that region due to occurrence of support reaction. Moreover, the appearance of higher plastic strain in $0.5 \mathrm{~mm}$ stepdown size as shown in figure 6(c) is mainly due to the close movement of tool motion between paths than $2.0 \mathrm{~mm}$ stepdown size configuration. Further, the stress fluctuations also because of the combination of both stretching and compression deformations during forming and besides, it is more helpful to notice that the stress is equal zero at the beginning, and then gradually increase to considerable forming distance and exhibited fluctuations before it reduces close to the tool retractable region. Again same as before, the milling process time happens to be increasing with respect to lower step down size in the normal direction as summarized in Table 3. 


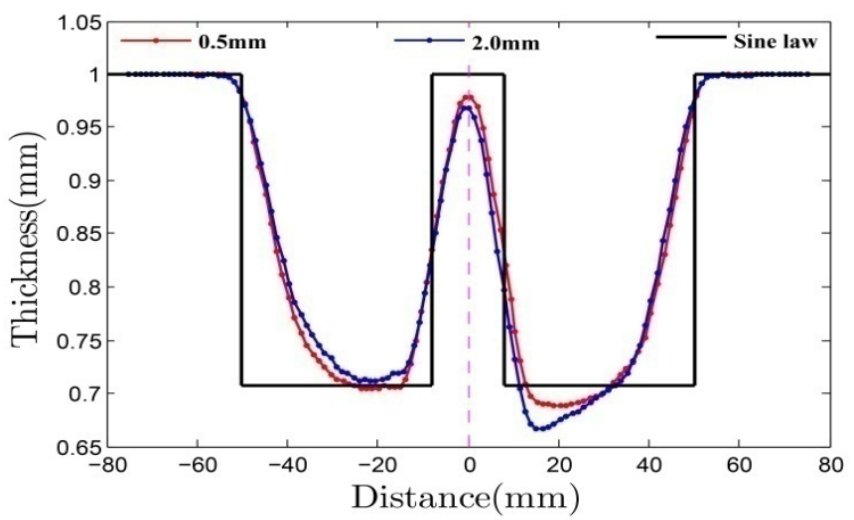

(a) Thickness Distribution (without BP)

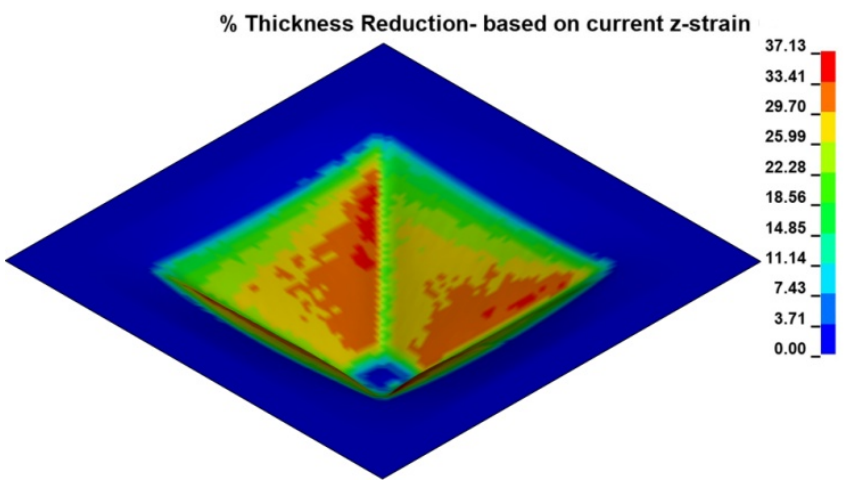

(c) Thickness Reduction in \% (without BP)

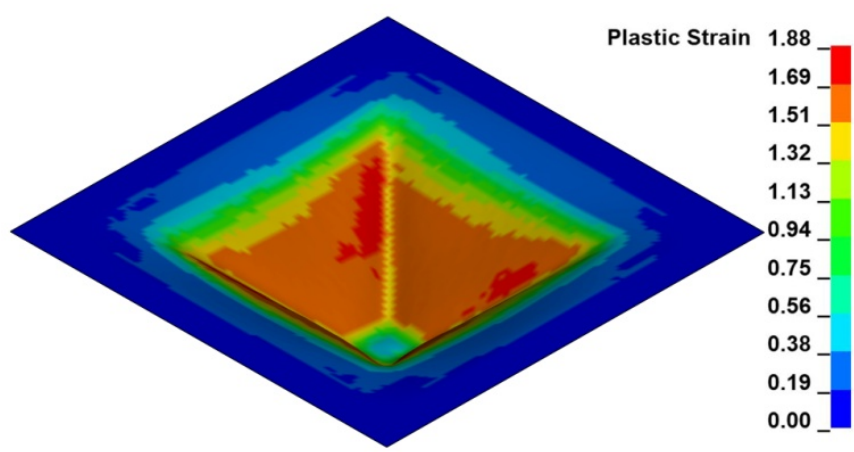

(e) Plastic Strain Contour (without BP)

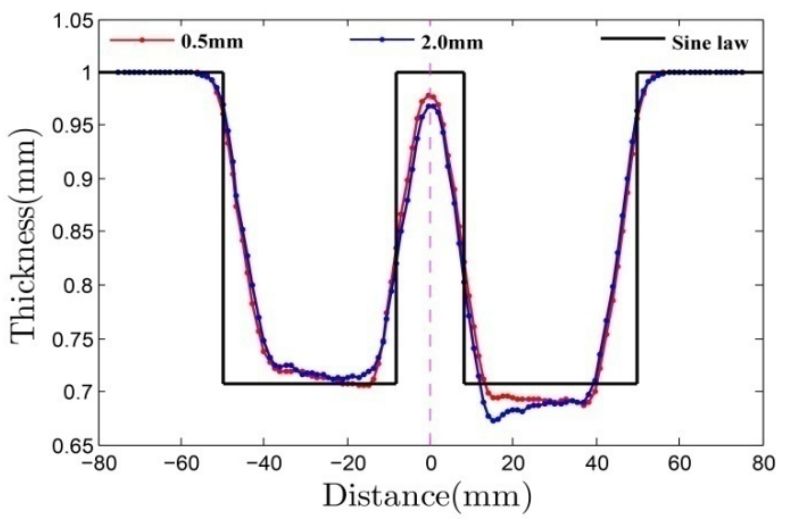

(b) Thickness Distribution (with BP)

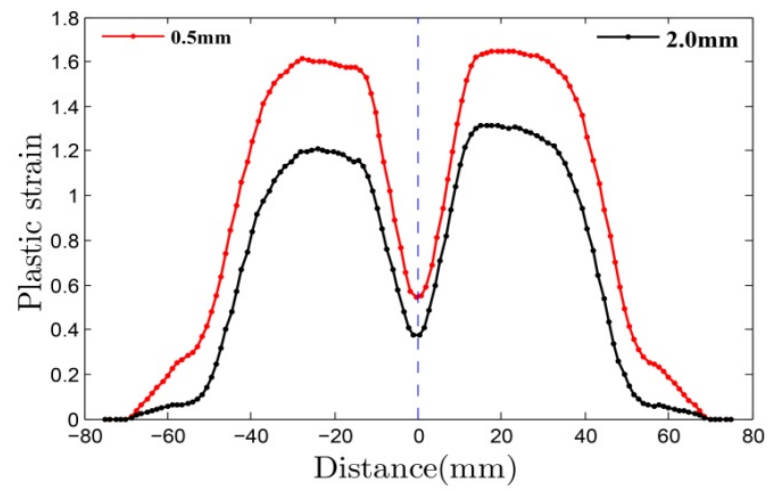

(d) Plastic Strain Distribution (without BP)

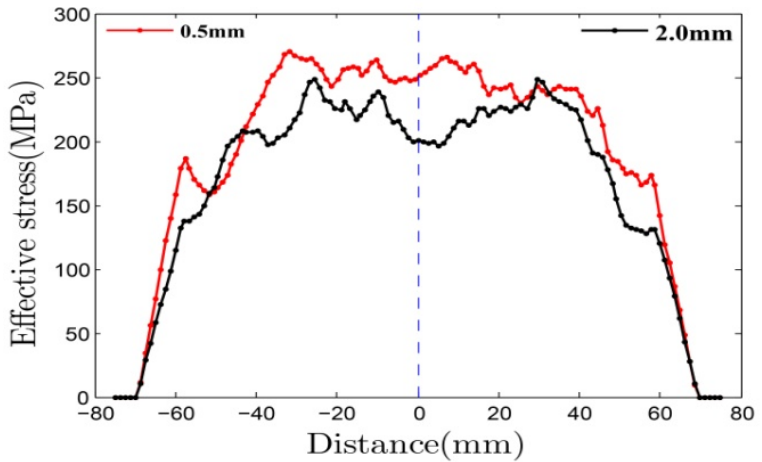

(f) Effective Stress Distribution (without BP)

Figure 6: SPIF Simulation Results of Pyramid Shape in Terms of Strain, Stress and Thickness Distributions.

Table 3: SPIF Numerical Conditions \& Results Comparison of Truncated Pyramid

\begin{tabular}{|c|c|c|c|c|c|}
\hline $\begin{array}{c}\text { Tool } \\
\text { Radius } \\
\end{array}$ & Feed Rate & Step Size & $\begin{array}{c}\text { Thickness } \\
\text { (Max) }\end{array}$ & $\begin{array}{c}\text { Thickness } \\
(\%)\end{array}$ & $\begin{array}{c}\text { Milling } \\
\text { Time }\end{array}$ \\
\hline \multirow{8}{*}{$2 \mathrm{~mm}$} & \multirow{8}{*}{$1000 \mathrm{~mm} / \mathrm{min}$} & \multirow{2}{*}{$0.5 \mathrm{~mm}$} & 0.6885 & 31.163 & \multirow{2}{*}{$20 \min 2 s$} \\
\hline & & & 0.6875 & 31.241 & \\
\hline & & \multirow{2}{*}{$1.0 \mathrm{~mm}$} & 0.6821 & 31.809 & \multirow{2}{*}{$10 \mathrm{~min} 11 \mathrm{~s}$} \\
\hline & & & 0.6822 & 31.773 & \\
\hline & & \multirow{2}{*}{$2.0 \mathrm{~mm}$} & 0.6665 & 33.348 & \multirow{2}{*}{$5 \min 15 \mathrm{~s}$} \\
\hline & & & 0.6727 & 32.754 & \\
\hline & & \multirow{2}{*}{$3.0 \mathrm{~mm}$} & 0.6679 & 33.217 & \multirow{2}{*}{$3 \mathrm{~min} 36 \mathrm{~s}$} \\
\hline & & & 0.6728 & 32.763 & \\
\hline
\end{tabular}


It is obvious that a complicated FE model is computationally more challenging in order to solve the constructed physical problem. To reduce computational time and cost, the model reduction techniques are commonly employed and in this research work, the desired shapes convey that SPIF numerical model can be reduced into the half model using symmetric boundary conditions. Therefore, the full numerical model was converted into the half model and the simulation was supervised applying generated contour path from $1.0 \mathrm{~mm}$ step down size and base plate configuration, as illustrated in Figure 7(a). The thickness variation from full model in comparison with half model implies that the half model has a capability to capture the thinning behaviour accurately by accounting the error of less than 1\%, as depicted in Figure 7(b). Also, the computational time was reduced from $12 \mathrm{hr} 33 \mathrm{~min}$ to $4 \mathrm{hr} 48 \mathrm{~min}$ and this outcome confirms the importance of reduced numerical model for solving SPIF process without compensating the accuracy on model measurements. Figure 7(c) benefits us to understand the fact that if the tool path step size is getting lower, the surface quality in terms of smooth finish tends to enhance as a result and vice versa.

Overall, comparison results from the numerical model measurements state that even though the CNC milling time is much higher while the step size is small, SPIF process can be successfully developed by adopting proper mesh counts, boundary conditions and mass scaling time settings. Also, the numerical model established in this research work can be devised for any products which show complexity in terms of manufacturing process.

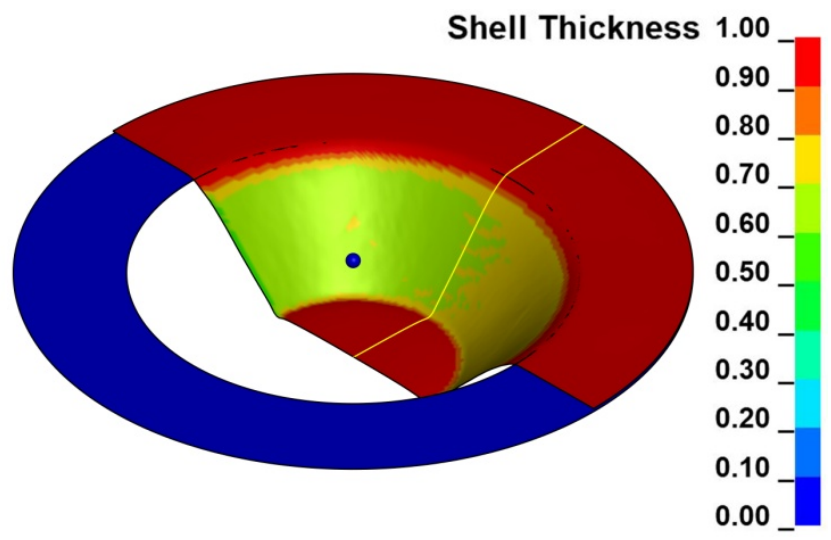

(a) Half Model (Symmetric Boundary Conditions)

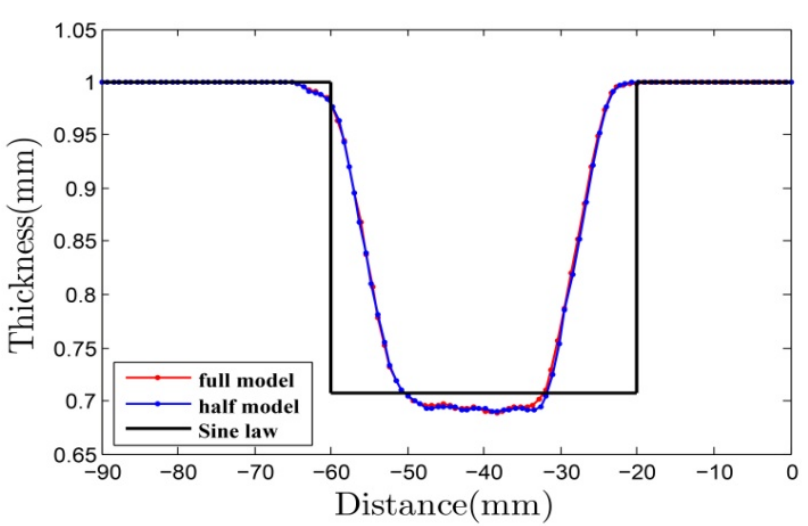

(b) Thickness Distribution (With BP)
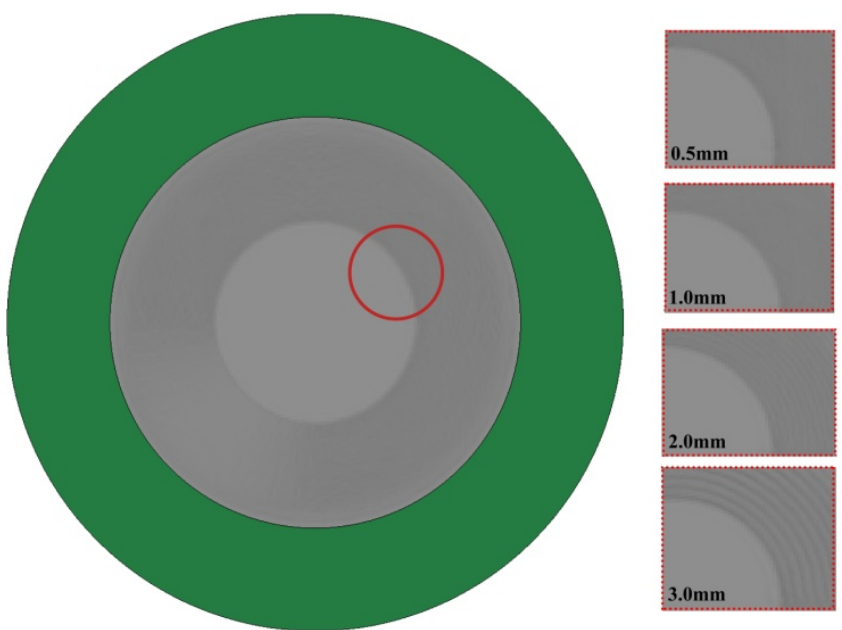

(c)Surface Quality Assessment

Figure 7: SPIF Numerical Results Of Half Truncated Cone Model. 


\section{CONCLUSIONS}

Numerical simulation of SPIF process was carried out for a truncated cone and a truncated pyramid with the wall angle of $45^{\circ}$ using aluminium alloy material properties. A comparison between simulation and desired design was made and based on the results, the following conclusions are organized. The desired shape accuracy was found to be quite low notably when a base plate configuration is not adopted in the process. Moreover thinning phenomenon occurs relatively higher during this process in the formed product. In addition, when the contour path step size was greater, the shape prediction accuracy was comparatively lower than the small step size contour path and the pillow effect was identified in the location that are not expected to be deformed. From plastic strain comparison between the formed products, the maximum plastic strain value was recognized when the contour path step size is minimum and it is because, the punch tool motion is closely tight at each path transition from one to another. The forming time was comparatively higher due to small step size against the shape accuracy. Moreover, for axisymmetric shapes, the computation time can be reduced with the help of symmetric model boundary conditions without affecting the results. Based on experience gained from this research, we conclude that SPIF process is well suited for producing complex shapes using simple punch tools and generated CAD/CAM tool paths.

\section{REFERENCES}

1. G. M. Reddy, P. Mastanaiah, C.V.S. Murthy, T.Mohandas and N. Viswanathan. Microstructure, Residual Stress Distribution and Mechanical Properties of Friction-Stir AA 6061 Aluminium Alloy Weldments. Indian Society for Non-Destructive Testing Hyderabad Chapter.

2. M.L. Gipiela, V. Amauri, C. Nikhare and P.V.P. Marcondes. A numerical analysis on forming limits during spiral and concentric single point incremental forming. IOP Conf. Series. Materials Science and Engineering, 2017, 164.

3. D.S. Malwad and V.M. Nandedkar. Deformation Mechanism Analysis of Single Point Incremental Sheet Metal Forming. $3^{\text {rd }}$ International Conference on Materials Processing and Characterization, Procedia Materials Science, 2014, 6, 1505-1510.

4. Manish Oraon and Vinay Sharma. Prediction of surface roughness in single point incremental forming of AA3003-O alloy using artificial neural network.Int. J. Materials Engineering Innovation, 2018, 9(1).

5. Suresh Kurra, N.H. Rahman, S.P. Regalla and A.K. Gupta. Modeling and optimization of surface roughness in single point incremental forming process. J. Mater. Res. Tech. 2015, 4(3), 304-313.

6. Erika Salem, Jaekwang Shin, Maya Nath, Mihaela Banu and Alan I. Taub. Investigation of thickness variation in single point incremental forming. Procedia Manufacturing, 2016, 5, 828-837.

7. D.M. Neto, J.M.P. Martins, M.C. Oliveira, L.F. Menezes and J.L. Alves.Evaluation of strain and stress states in the single point incremental forming process. Int. J. Adv. Manuf. Tech. 2016, 85, 521-534.

8. Blaga, O. Bologa, V. Oleksik and R. Breaz. Influence of tool path on main strains, thickness reduction an forces in single point incremental forming process. Proceedings in Manufacturing Systems, 2011, 6(4).

9. J. Jeswiet, J.R. Duflou and A. Szekeres. Forces in Single Point and Two Point Incremental Forming. Advanced Materials Research, 2005, 6-8, 449-456.

10. Patel, V. A. Vertical Turbine Pump base Stool Modal Analysis and Model Modification. 
11. H. Markanday and D. Nagarajan. Formability behaviour studies on CP-Al sheets processed through the helical tool path of incremental forming process. IOP Conf. Series. Materials Science and Engineering, 2018, 314.

12. D.D. Storkle,D. Mollensiep, L. Thyssen and B. Kuhlenkotter. Geometry-dependent parameterization of local support in robotbased incremental sheet forming. $17^{\text {th }}$ International Conference on Metal Forming, Procedia Manufacturing, 2018, 15, 11641169.

13. J. Naranjo, V. Miguel, A. Martinez-Martinez, L.M. Gomez-Lopez, M.C.Manjabacas and J. Coello. Analysis and simulation of Single Point Incremental Forming by ANSYS. The Manufacturing Engineering Society International Conference, Procedia Engineering, 2015, 132, 1104-1111.

14. Chikalthankar, S. B., Nandedkar, V. M., \& Borde, S. V. (2013). Influence of Machining Parameters on Electric Discharge Machining of WPS Tool Steels-An Experimental Investigation. International Journal of Mechanical and Production Engineering Research and Development (IJMPERD), 3(5), 21-28.

15. P. Memicoglu O. Music and C. Karadogan. Simulation of incremental sheet forming using partial sheet models. International Conference on Technology of Plasticity. Procedia Engineering. 2017, 207, 831-835

16. Matsubara. S. Incremental backward bulge forming of a sheet metal with a hemispherical head tool-a study of a numerically controlled forming system II. J. Jpn. Soc. Tech. Plastic.1994, 35 (406), 1311-1321.

17. Young. D and Jeswiet. J. Wall thickness variations in single-point incremental forming. J. Eng. Manufact. Part B, 2004, 218, 1453-1459.

18. Ambrogio. G, Filice. L, Gagliardi. F and Micari. F. Sheet thinning prediction in single point incremental forming. International Conference on Sheet Metal, 2005, 479-486.

19. Ali, A. A. M., Negm, A., Bady, M., \& Ibrahim, M. G. E. (2014). Towards an integrated tool to estimate carbon emissions from life cycle assessment of building materials in Egypt. International Journal of Research in Engineering and Technology, 2(3), $81-92$.

\section{AUTHOR PROFILE}

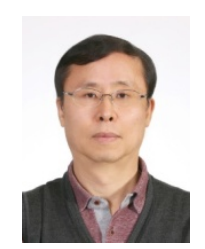

Professor Dong-Won Jungwas born in Seoul, South KOREA on February 23, 1964. He received the Master degree and the Ph.D. degree in mechanical engineering from KAIST, Daejeon, South KOREA, in 1991 and 1995, respectively. He is currently a Professor in the Department of Mechanical Engineering, Jeju National University, and has been the Director of the Laboratory of Computational Solid Mechanics, Jeju National University, since 1997. From 2006 to 2008, he was the Director of the factory of Jeju National University. Since 2002, he has been the Dean of the Faculty of Mechanical Engineering, Jeju National University. He is a member of KSPE, IJPEM, IJPEM-GT, KSME, KSTP, etc. He has published more than 250 scientific papers in the field of metal forming, FEM analysis, and design developments. His research interests include the die development, structure analysis, impact analysis, optimization, polymer and composite materials, etc. 


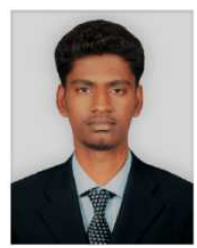

Mohanraj Murugesan was born in Unjini, Tamil Nadu,India, in 1992. He received his bachelor's degree (BE) in department of Aeronautical engineering from Kumaraguru College of Technology (KCT)/Anna University in 2009-2013. After completing his course from KCT, in February 2014, he joined at Pusan National University (PNU) to purse his master's course (MSc) in school of aerospace engineering from 2014-2016.After graduating from PNU with master's degree (first class) he head for a $\mathrm{PhD}$ in mechanical engineering. He then spent some valuable time in Sogang University and gained research experience in various topics related to metal forming. From 2019 February, he enrolled at Jeju National University (JNU) for his PhD degree in mechanical engineering. He joined Professor Dong-Won Jung lab for his $\mathrm{PhD}$ research. These days he is working on flow stress prediction under hot working conditions, microstructure evaluation (FESEM/EDS) in the work material before and after testing/forming, stamping/deep drawing, cold roll forming and single point incremental sheet forming process (experimental and numerical). He has extensive experience on design, numerical modeling, programming and experimental methods. He has more than 20 research publications including both conference and journal.

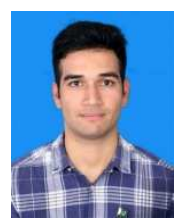

Muhammad Sajjad was born in Shawa Dir Lower KPK, Pakistan, in 1994. He received his B.Sc degree in Mechanical Engineering from Swedish College of Engineering \& Technology WahCantt Pakistan, in 2017. In March 2018, he joined the Jeju National University as a Master student, currently enrolled in the last semester of his master degree. He joined Professor Dong-Won Jung lab for his master research. In lab currently he is working on roll forming and incremental forming of aluminum alloys. He has good experience of the use of design and simulation software which currently he is using for his research study in his master degree. He has more than 12 journal publication which includes few conference proceeding publications. 